\title{
META-MODEL ASSISTED MULTI-OBJECTIVE OPTIMIZATION FOR NON-STEADY 3D METAL FORMING PROCESSES
}

\author{
M.Ejday*, L.Fourment, \\ Mines Paris Tech, CEMEF-Centre for Material Forming, CNRS UMR 7635, BP 207, 1 rue \\ Claude Daunesse, 06904 Sophia Antipolis Cedex, France
}

\begin{abstract}
This paper studies efficient techniques to find the optimal set of solutions (Pareto front) for multiobjective optimization problems in the context of time expensive evaluation of functions. These techniques make use of a meta-model based on the Meshless Finite Difference Method (MFDM) coupled with evolutionary Multi-Objective algorithm (here: NSGA-II) in order to minimize the time consuming evaluations and to achieve a faster convergence to the Pareto front. The different studied methods differ in the choice of master points, the evolution of the meta-model, and the updating of elitism. They are studied and compared on several analytical functions, with only 100 exact evaluations of the objective function. The obtained results show the efficiency of these techniques.
\end{abstract}

KEYWORDS: Meta-model, Evolutionary Algorithms, Multi-Objective Optimisation, MFDM, NSGA-II

\section{INTRODUCTION}

Multi-objective Evolutionary Algorithms (MOEA) are the best techniques for determining the Pareto front of Multi-Objective Optimisation Problems (MOOP). They are aiming at detecting a good diversity of points on the Pareto set for difficult problems. However they require a large number of function evaluations, which sometimes makes these strategies impracticable for problems with time consuming evaluations. The number of expensive function evaluations can be significantly reduced by partly replacing precise evaluations by fast approximate models of the objective functions. This is done in the proposed Meta-model assisted MOEA (M-MOEA) by introducing three different manners to update the metamodel inside the MOEA, before applying it to analytical functions for evaluating their efficiency.

\section{MULTI-OBJECTIVE OPTIMISATION PROBLEM (MOOP)}

A Multi-Objective Optimization Problem (1) implies a number of $M$ objective functions $\phi_{m}(X)$ which are to be either minimized or maximized with respect to $X$, a vector of $n$ decision variables: $X=\left(x_{1}, x_{2}, \ldots, x_{n}\right)^{T}$, where $n$ is the number of parameters. The MOOP usually has a number of constraints which any feasible solution must satisfy, so constituting the decision space $S$.

$$
\text { Minimize/Maximize } \phi_{m}(X) \quad ; \quad m=1, \ldots, M
$$

The presence of multiple conflicting objectives gives a set of trade-off optimal solutions that can found by the concept of domination [1-4]: a solution $X^{l}$ is said to dominate another solution $X^{2}$, if the following two conditions are true:

1. solution $X^{l}$ is not worse than $X^{2}$ for all $M$ objectives.

2. solution $X^{l}$ is strictly better than $X^{2}$ for at least one objective.

The set of non-dominated solutions in $S$ is known as Pareto set and its graphic representation as the Pareto optimal front. Two goals are to be achieved in MOOP: 1) finding a set of solutions close to the Pareto front, 2) selecting a set that is diverse enough to represent the spread of the front.

\section{ELITIST NON-DOMINATED SORTING GENETIC ALGORITHM (NSGA-II)}

NSGA-II is an elitist Non-dominated Sorting Genetic Algorithm developed by Deb [1,2], which is more precisely described in $[3,4]$, and which is one of the most efficient algorithm for finding Pareto optimal sets with good diversity of solutions. It has the following three characteristics:

1. it uses an elitist principle,

2. it emphasizes non dominated solutions,

3. it uses an explicit diversity of solutions.

The NSGA-II starts with a randomly generated initial parent population $P_{0}$ of $N$ individuals. At the generation $t$, the population $Q_{t}$ of the $N$ children is first created from the $P_{t}$ parent population using the usual genetic operators (selection, crossover, mutation). Thereafter, the two populations are combined together to form the $R_{t}$ set of size $2 N$. A non dominated sorting is carried out to classify its individuals by several fronts of various ranks.

\footnotetext{
* Corresponding author: Mohesen Ejday, Mines ParisTech, CEMEF, CNRS UMR 7635, 1 rue Claude Daunesse, 06904 Sophia Antipolis, France, phone: +33 (0)4 9395 75 75, fax: +33 (0)4 923897 52, mohesen.ejday@mines-paristech.fr
} 
These sets are obtained iteratively. For any unclassified individual, a pair comparison is made with all previously classified ones through the concept of domination. The individuals that are not dominated by any other one compose the first rank of the Pareto front. By temporarily discarding these individuals, the algorithm can be repeated again to find the next non dominated front. Then, the new population $P_{t+1}$ is constituted by individuals of the different non dominated fronts of increasing order. The affectation starts with the best non dominated front (front of rank one), followed by the second rank, and so on. As the total size of the new parent population $P_{t+1}$ is $N$., not all fronts can be included in it, but only the ones having the smaller ranks. In the last possible front, there are usually more solutions than the remaining places in the new population. Therefore, the individuals of this last front are sorted in descending order of the crowding distance [1], and those with larger distances are accepted. With this method, the selected solutions are well scattered in the last allowed front.

The main disadvantage of Evolutionary Algorithms are their computational cost in terms of number of function evaluations, which is generally in the magnitude of several thousands. To overcome this shortcoming, in the frame of applications to metal forming problems requiring several hours of computational time on a parallel machine for a single function evaluation, a metamodel is developed.

\section{META-MODEL}

The selected meta-model is based on the Meshless Finite Difference Method (MFDM) [5,6]. The approximated value $\tilde{\phi}_{i}$ of $\phi\left(X_{i}\right)$ is calculated for any point $i$ located at $X_{i}$ in the design space, from the $n_{m} j$ values, $\phi_{j}=\phi\left(X_{j}\right)$, that are known at the so-called master points $j$, by writing the first order Taylor series expansions at $i$ for any $j$ point $\widetilde{\phi}_{i}$ and its gradient $\nabla \tilde{\phi}_{i}$ being unknown (see equation (2)). The solution $\left(\tilde{\phi}_{i}, \nabla \tilde{\phi}_{i}\right)$ minimizes the cumulated error $E\left(\tilde{\phi}_{i}, \nabla \tilde{\phi}_{i}\right)$ (see equation (3)) for all the $n_{m}$ considered master points, which results into the resolution of a linear system (4).

$$
\begin{gathered}
\forall j=1, n_{m}, \quad \phi_{j}=\widetilde{\phi}_{i}+\nabla \widetilde{\phi}_{i}\left(X_{j}-X_{i}\right)+O\left(\left\|X_{j}-X_{i}\right\|^{2}\right) \\
E\left(\widetilde{\phi}_{i}, \nabla \widetilde{\phi}_{i}\right)=\frac{1}{2} \sum_{j=1, n_{m}} \frac{\left(\widetilde{\phi}_{i}+\nabla \widetilde{\phi}_{i}\left(X_{j}-X_{i}\right)-\phi_{j}\right)^{2}}{\left\|X_{j}-X_{i}\right\|^{4}} \\
A\left(\begin{array}{c}
\widetilde{\phi}_{i} \\
\nabla \widetilde{\phi}_{i}
\end{array}\right)=b\left(\left(\phi_{j}\right)_{j=1, n_{m}}\right)
\end{gathered}
$$

The interpolation error $\Delta \widetilde{\phi}_{i}$ of $\widetilde{\phi}_{i}$ at point $i$ can be estimated by:

$$
\Delta \widetilde{\phi}_{i}=\sqrt{2\left(\sum_{j=1, n_{m}} \frac{1}{\left\|X_{j}-X_{i}\right\|^{4}}\right)^{-1} E\left(\tilde{\phi}_{i}, \nabla \widetilde{\phi}_{i}\right)}
$$

\section{META-MODEL ASSISTED NSGA-II}

In this section, we present three successive approaches to introduce the MFDM meta-model into the NSGA-II.

\subsection{CONSTANT M-MOEA: M $_{0}$-NSGA-II}

In the $\mathrm{M}_{0}$-NSGA-II, the NSGA-II first generates the initial population $P_{0}$ of size $N(=100)$. All these individuals, that are randomly and hopefully uniformly distributed in the designed space, are regarded as the $\lambda$ master points $(\lambda=100)$ of the $M_{0}$ constant metamodel. The $P_{0}$ population is then exactly evaluated, but in the next generations, the offspring population $Q_{t}$ is only approximately evaluated by using the meta-model $M_{0}$ (equations (2-4) with $n_{m}=\lambda$ ).

\subsection{UPDATED M-MOEA: $M_{1}$-NSGA-II}

In the $\mathrm{M}_{1}$-NSGA-II approach, the initial meta-model $M_{0}$ is constructed by using only the $\lambda \leq \lambda_{\max }=100$ first individuals of the population $P_{0}$. Then, $M_{0}$ is used to approximate the objective function values for the remaining individuals of $P_{0}$. At each new generation $t$, the $M_{t}$ meta-model is iteratively enriched into $M_{t+1}$ by adding $\theta$ new master points, which are selected as the $\theta$ first individuals of the $Q_{t}$ offspring population. $\theta$ is calculated by equation (6), as a function of the prescribed number of generations, $N_{g}$.

$$
\theta=\frac{\lambda_{\max }-\lambda}{N_{g}}
$$

The new meta-model $M_{t+1}$ is used to estimate the objective functions of the $Q_{t}$ population as well as to update the estimations of the $P_{t}$ parent population, which was carried out with the $M_{t}$ meta-model.

\subsection{IMPROVED M-MOEA: $\mathrm{M}_{\mathrm{e}}$-NSGA-II}

The improved M-MOEA, Me-NSGA-II, only differs from $\mathrm{M}_{1}$-NSGA-II by the way of selecting the $\theta$ new master points of $Q_{t}$. The values of the objective functions of any $Q_{t}$ individual are first approximated by $(\tilde{\phi}-\Delta \widetilde{\phi})$, which represents the best expected value of any individual taking into account the approximation error, using the $M_{t}$ meta-model. Then, the $Q_{t}$ individuals are classified according to their rank and 
crowding distance. The $\theta$ first are selected to enhance $M_{t}$ into $M_{t+1}$.

\section{ANALYTICAL PROBLEMS}

In this section, the results obtained by $\mathrm{M}_{0}$-NSGA-II and $\mathrm{M}_{1}$-NSGA-II for a wide range of analytical problems are presented and compared to those provided by the NSGAII, where all functions are computed exactly. The algorithm uses a population of $N=100$, and a number of generation $N_{g}$ equals to 50 .

\subsection{TEST FUNCTION: Min-Ex}

For the M-MOEA, we set $\lambda_{\max }=100, \lambda=40, N_{g}=40, \theta=2$. The Min-Ex function proposed by Deb [1] is given by:

$$
\text { Min-Ex:\{ } \begin{array}{ll}
\text { Minimize } & f_{1}\left(x_{1}, x_{2}\right)=x_{1} \\
\text { Minimize } & f_{2}\left(x_{1}, x_{2}\right)=\frac{1+x_{2}}{x_{1}} \\
\text { subject to } & 0.1 \leq x_{1} \leq 1 ; 0 \leq x_{2} \leq 5
\end{array}
$$

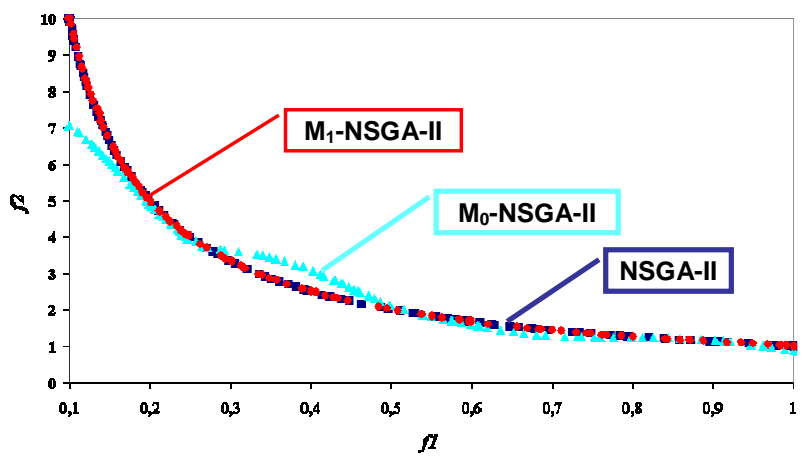

Figure 1: Comparison of the Pareto front of Min-EX obtained by Mo-NSGA-II, M1-NSGA-II and NSGA-II

Figure 1 and 2 show that with only 100 function evaluations, $\mathrm{M}_{0}$-NSGA-II provides a good approximation of the Pareto front that is quite correctly obtained by NSGA-II with 5,000 function evaluations. However, there are some discrepancies in two zones, which are due to the fact that the constant meta-model is not accurate enough in the vicinity of the Pareto front. This shortcoming is corrected by the $\mathrm{M}_{1}$-NSGA-II approach that allows constantly improving the metamodel. As the algorithm converges toward the Pareto front, the newly added master points are also closer to this front and the approximation is consequently improved.

\subsection{TEST FUNCTION: SCH2}

$\mathrm{SCH} 2$ (see equation (8)) is a test problems proposed by Schaffer that exhibit a discontinuous Pareto front. For the M-MOEA, we set $\lambda_{\max }=100, \lambda=10, N_{g}=45, \theta=2$.

$$
\begin{gathered}
\text { Minimize } f_{1}(x), f_{2}(x) \\
f_{1}(x)=\left\{\begin{array}{cl}
-x & \text { if } x \leq 1 \\
x-2 & \text { if } 1<x \leq 3 \\
4-x & \text { if } 3<x \leq 4 \\
x-4 & \text { if } x>4
\end{array}\right. \text {. }
\end{gathered}
$$

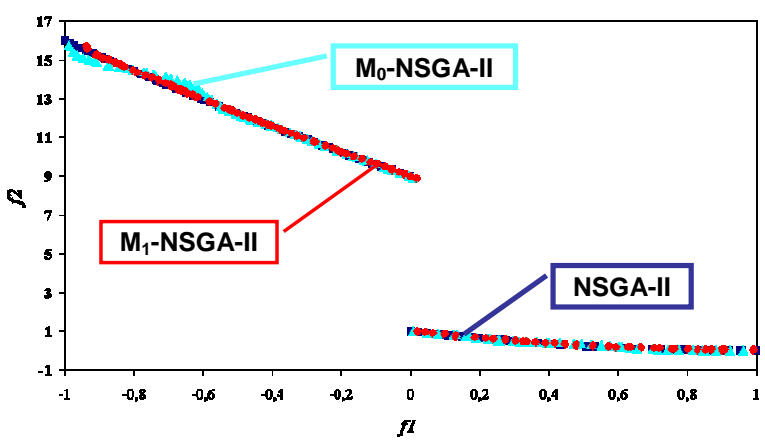

Figure 2: Comparison of the Pareto fronts of $\mathrm{SCH} 2$ obtained by $M_{0}-N S G A-I I, M_{1}-N S G A-I /$ and NSGA-II

Figure 2 shows that both M-MOEA allow accurately describing the discontinuous Pareto front with a limited number of function evaluations, although $\mathrm{M}_{1}$-NSGA-II is slightly more accurate than $\mathrm{M}_{0}$-NSGA-II.

\subsection{TEST FUNCTION: FON}

The FON functions (see equations (9)) proposed by Fonseca and Fleming provide a multi-dimensional problem with $n$ variables (here $n=2$ ). The NSGA-II algorithm requires 250 generations to find an appropriate Pareto front. For the M-MOEA, we set $\lambda_{\max }=100, \lambda=20$, $N_{g}=20, \theta=4$ and keep the total number of function evaluations to 100 .

$$
\begin{gathered}
\operatorname{Minimize} f_{1}(x)=1-\exp \left(-\sum_{i=1}^{n}\left(x_{i}-\frac{1}{\sqrt{n}}\right)^{2}\right) \\
\text { Minimize } f_{2}\left(x=1-\exp \left(-\sum_{i=1}^{n}\left(x_{i}+\frac{1}{\sqrt{n}}\right)^{2}\right)\right. \\
-4 \leq x_{i} \leq 4
\end{gathered}
$$

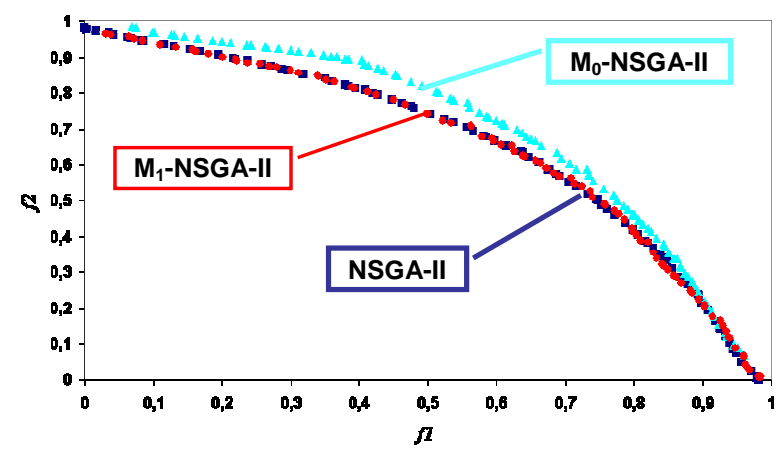


Figure 3: Comparison of the Pareto fronts of FON obtained by $M_{0}-N S G A-I I, M_{1}-N S G A-I /$ and NSGA-II

The obtained results (see Figure 3) are quite similar to those of section 6.1, even though the FON functions are more complex and the standard NSGA-II encounters more difficulties to find the Pareto front.

\section{METAL FORMING PROBLEM}

This first metal forming application regards the shape optimization of a cylindrical billet in order to forge a connecting rod. The MOOP (see equation 10) consists in minimizing the preform diameter, $\mathrm{x}$, while maximizing the filling of the dies at the end of forging (see Figure 4), in order to obtain the proper component final shape.

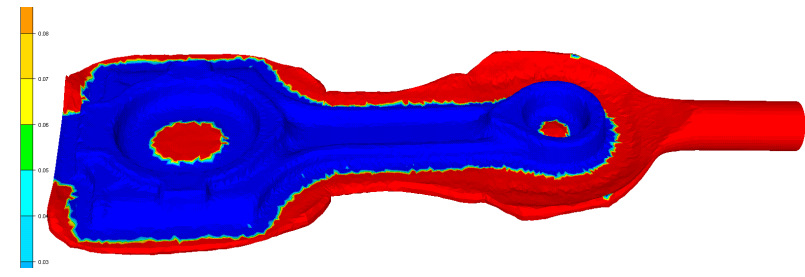

Figure 4: Forging of a connecting rod with the Forge $3 \AA$ software: isovalues of the distance between the workpiece and the dies (blue $\rightarrow$ proper filling)

$$
\left\{\begin{array}{c}
f(x) \text { : minimize filling \& minimize volume } \\
0.8<x<1.05
\end{array}\right.
$$

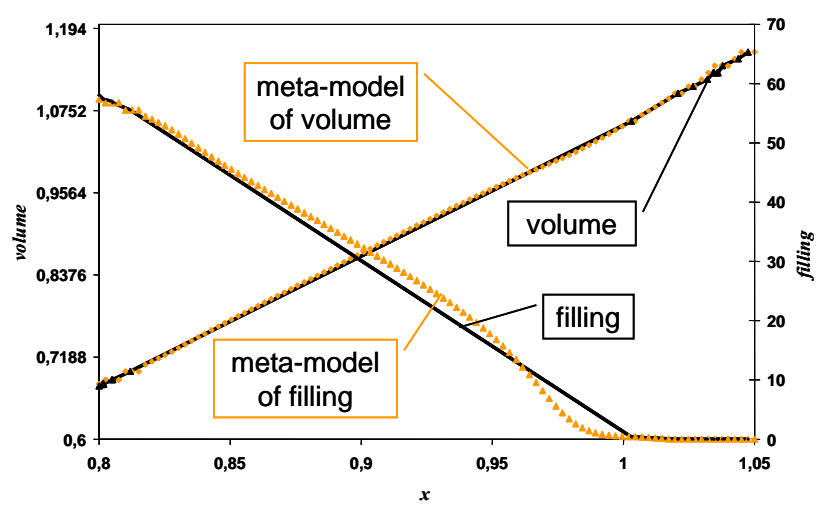

Figure 5: Meta-models of the "filling" and "volume" functions versus the cylinder diameter $x$.

By using weights and combining the two objective functions, the problem is first regarded like a single objective optimization problem. A meta-model assisted evolutionary algorithm makes it possible to obtain an interesting solution (proper filling with reduced weight of the initial billet) within only 20 actual forging simulations. These results are regarded like master points to build meta-models of the objective functions (Figure 5).

In a second step, the meta-models of Figure 5 are used within the $\mathrm{M}_{0}$-NSGA-II algorithm to compute the Pareto front of the MOOP (see Figure 6), which so provides a wider range of possible solutions according to the requirements on the filling accuracy.

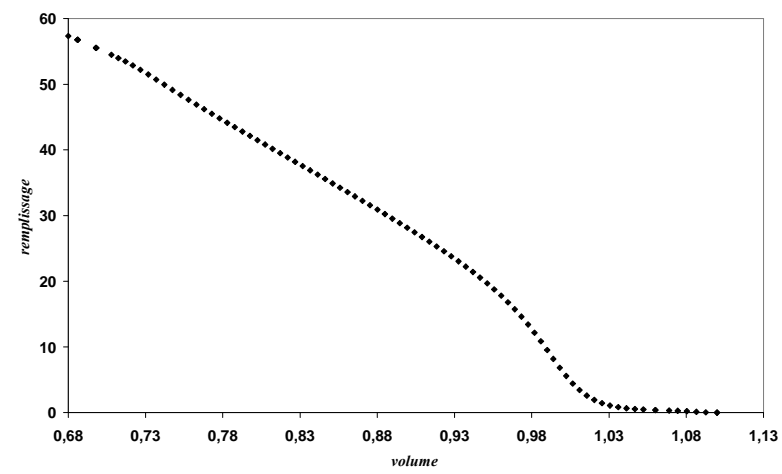

Figure 6: Pareto front of the metal forming problem.

\section{CONCLUSION}

This study shows that M-MOEA are able to properly approximate Pareto fronts for a wide range of analytical problems with convex (Min-Ex), concave (FON) and discontinuous ( $\mathrm{SCH} 2)$ fronts within a limited number of function evaluations (100). It shows that it is very important to update the meta-model during the algorithm iterations to properly approximate the objective functions in the vicinity of the Pareto front.

\section{ACKNOWLEDGEMENT}

This work has been carried out in the frame of the LOGIC ANR program, which support is gratefully acknowledged.

\section{REFERENCES}

[1] Deb.K.: Multi-Objective Optimisation using Evolutionary Algorithms. John Wiley \& Sons Chichester, 2001.

[2] K. Pawan, S.Nain, and K. Deb. A Multi-Objective Optimization Procedure with Successive Approximate Models. KanGAL Report Number 2005002.

[3] M. Emmerich and B. Naujoks. Metamodel-Assisted Multiobjective Optimization with Implicit Constraints and its Application in Airfoil Design. In International Conference \& Advanced Course ERCOFTAC, Athens, Greece, 2004.

[4] T. Goal, R. Vaidyanathan, R. T. Haftka, W. Shyy, N. V. Queipo, and K. Tucker. Response surface approximation of Pareto optimal front in multiobjective optimization. Comput. Methods Appl. Mech. Engrg, 196, 879-893, 2007.

[5] T. Liszka, J. Orkisz. The finite difference method at arbitrary irregular grids and its application in applied mechanics. Comp.and Struct 11, 83-95, 1980.

[6] M. T. T. Do. Optimisation de forme en forgeage 3D. Ph. D. thesis, Mines ParisTech, 2006 